\title{
Plasma levels of matrix metalloproteinase-2 and -9 (MMP-2 and MMP-9) in age- related macular degeneration
}

KY Chau', S Sivaprasad², N Patel', TA Donaldson', PJ Luthert' and NV Chong ${ }^{1,2}$

\begin{abstract}
Background Several studies indicate that agerelated macular degeneration (AMD) and atherosclerosis may share common pathogenetic pathways. The aim of this study was to determine the role of systemic matrix metalloproteinases (MMPs) in AMD, given that MMPs are implicated in the pathogenesis of atherosclerosis.

Methods This study determined the plasma matrix metalloproteinases (MMP-2 and MMP9) levels in three groups of subjects: group 1 included subjects with age-related maculopathy (ARM), group 2 included subjects with choroidal neovascularization (CNV) owing to AMD and group 3 consisted of age-matched controls.

Results The mean plasma levels of MMP-2 were not significantly different in the three groups. In contrast, the mean plasma MMP-9 levels were significantly higher in ARM and CNV groups compared to that of the control group. However, there was no significant difference in MMP-9 levels between ARM and CNV groups. Conclusion This is the first study that reveals a link between raised plasma MMP-9 levels with AMD. Further studies are required to identify the factors that contribute to this association.

Eye (2007) 21, 1511-1515; doi:10.1038/sj.eye.6702722; published online 16 February 2007
\end{abstract}

Keywords: MMP-2; MMP-9; age-related macular degeneration

\section{Introduction}

Age-related macular degeneration (AMD) is a leading cause of blindness in the elderly. ${ }^{1-3}$
Early stage of the disease is characterized by drusen and retinal pigmentary changes and in this article it is termed as agerelated maculopathy (ARM). Fifteen percent of the affected individuals experience profound loss of central vision owing to the development of choroidal neovascularization (CNV). ${ }^{4}$

The data-linking aberrations in extracellular matrix (ECM) biology and AMD are mounting. ${ }^{5,6}$ Numerous histopathological studies have shown that ECM components such as the collagen and elastic layers in Bruch's membrane undergoes substantial changes in AMD. ${ }^{7,8}$

Matrix metalloproteinases (MMPs) are a family of zinc-dependent endopeptidases that degrade substrates such as elastin, gelatine and collagen I, IV and V. These enzymes are expressed constitutively but under pathological states their expression can be increased. An accumulation of MMP-2 and MMP-9 has been identified in the Bruch's membrane of AMD eyes, suggesting the presence of a pathologic local enzymatic process. ${ }^{9,10}$ In addition, cellculture studies have documented the effect of MMP-2 and MMP-9 in the development of CNV. ${ }^{11,12}$

Compositional similarity between drusen and atherosclerotic plaques lend support to the theory that AMD and atherosclerosis may be parallel tissue responses to abnormal ECM turnover. ${ }^{13}$ MMPs have been implicated in atherosclerosis. Among them, increased circulating MMP-9 (gelatinase-B) has been identified as a predictor of cardiovascular mortality in patients with coronary artery disease. ${ }^{14}$ Likewise, MMP-2 (gelatinase-A) plays a pivotal role in oxidized low-density
${ }^{1}$ Institute of Ophthalmology, University College, London, UK

${ }^{2}$ Laser and Retinal Research Unit, King's College Hospital, Denmark Hill, London, UK

Correspondence: NV Chong, Laser and Retinal Research Unit, King's College Hospital, Denmark Hill, London, SE5 9RS, UK Tel: + 442073464548 ; Fax: + 442073463738

E-mail: victor@

eretina.org

Received: 9 March 2006 Accepted in revised form: 7 June 2006

Published online: 16

February 2007

Commercial relationships: None. 
lipoproteins (oxLDL) induced smooth muscle cell proliferation involved in atherosclerosis. ${ }^{15}$

Since systemic MMP-9 and MMP-2 are associated with atherosclerosis and localized MMP-2 and MMP-9 are associated with CNV secondary to AMD, we hypothesize that if atherosclerosis and AMD share a common mechanism, systemic MMP-9 and MMP-2 may be increased in patients with AMD.

\section{Materials and methods}

\section{Subjects}

This research adhered to the tenets of the Declaration of Helsinki. Institutional Ethics Committee approval was obtained and all subjects gave their full informed consent. The subjects in this study were recruited as part of a Medical Research Council (UK) funded project on AMD.

Thirty-three subjects with varying grades of AMD were recruited for the study from the Macula Clinic of King's College Hospital, London. Participants of the control group $(n=17)$ were selected among subjects awaiting pre-assessment for cataract surgery who were 50 years or older and fundus examination revealed the absence of drusen, pigmentary abnormalities and late AMD. Subjects with coexistent fundus pathology and subjects with ungradable photographs were excluded. The study did not include any subject with a history of neoplastic, hepatic, infectious or autoimmune disease; or any surgical procedure in the preceding 6 months.

All enrolled subjects underwent a complete ophthalmic examination by the recruiting retinal specialist (NVC): visual acuity, slit lamp examination and retinal examination after pupil dilation were documented. Each subject had $35^{\circ}$ colour stereo fundus photographs of both eyes taken (Topcon TRC 50IX, Topcon Ltd, Tokyo, Japan). Fluorescein angiography was performed if there was a clinical suspicion of CNV. A detailed medical history of each patient was taken with particular care taken to note history of cardiovascular disease including coronary artery disease, stroke and peripheral arterial disease.

\section{Grading of AMD}

Fundus photographs of these subjects were randomized and graded by two retinal specialists (NP, SS) using the nomenclature and classification recommended by the International ARM Epidemiological Study Group. ${ }^{16}$ The graders were masked of the age and clinical history of the participants. Double grading for intra-observer and inter-observer variability was performed. Discrepancies were resolved by discussion.
Briefly, we classified the subjects into three groups: (a) control group consisted of subjects with no evidence of drusen, retinal pigmentary abnormalities or late AMD; (b) ARM group included subjects with the presence of either large $(\geqslant 63 \mu \mathrm{m})$ soft distinct drusen with pigmentary abnormalities, or indistinct drusen ( $\geqslant 125 \mu \mathrm{m}$ ) or reticular drusen); and (c) CNV group included subjects with fluorescein angiographic evidence of CNV. If the grades in the two eyes were different, the subject was categorized according to the severity of changes in the worse eye.

\section{Plasma collection}

Venous samples were collected into Heparin tubes (Vacutainer, BD Diagnostics, Oxford, UK), centrifuged at $1000 \mathrm{~g}$ for $15 \mathrm{~min}$ at room temperature and plasma was transferred to a fresh tube within an hour of collection and kept frozen at $-80^{\circ} \mathrm{C}$. Plasma samples were thawed on ice and used for MMP measurements. The samples were randomized so that the scientist who analysed the samples was blinded of the clinical history of the subjects.

\section{Measurement of total plasma protein concentration}

The Coomassie Blue Protein Assay reagent (Pierce, Rockford, IL, USA) was used to determine the total protein level of each plasma sample. Any sample with reading that was significantly different from the average value was excluded.

\section{Measurement of total plasma MMP-2 concentration}

Plasma MMP-2 levels were quantified with a sandwich enzyme-linked immunosorbent assay (ELISA) using the Quantikine total human MMP-2 kit (R\&D Systems Inc., Minneapolis, MN, USA). The kit assayed the proactive and active forms of the MMP-2. The assays were processed according to the manufacturer's instruction.

All samples were measured in duplicate and in three serial dilutions and the results were averaged as mean \pm s.e.m.

\section{Measurement of total plasma MMP-9 concentration}

Plasma MMP-9 levels were also quantified with a sandwich ELISA using the Quantikine total human MMP-9 kit (R\&D Systems Inc.). The kit assayed the proactive and active forms of the MMP-9. The assays were processed according to the manufacturer's instructions. 
All samples were measured in duplicate and in three serial dilutions and the results were averaged as mean \pm s.e.m.

\section{Statistical methods}

MMP-2 and MMP-9 levels in the three groups were analysed using Student's $t$-test. The one-way ANOVA was also used to identify the relationship between patient parameters. Statistical significance was set as $P<0.05$.

\section{Results}

\section{Characteristics of the subjects}

Plasma was analysed from 50 subjects consisting of 15 with ARM, 18 with CNV and 17 controls. There were no significant differences in average age, gender distribution or prevalence of major risk factors among the three groups (Table 1).

\section{Quality of plasma samples}

Total protein measurements were performed as a preliminary test for the quality of plasma samples collected. Statistical analysis showed that there was no significant difference $(P>0.05)$ in the protein level of the samples between subjects in the three identified groups. The average measurement is $3.3 \pm 0.3 \mathrm{mg} / \mathrm{ml}$.

\section{Total plasma MMP-2 levels}

The mean plasma levels of MMP-2 in control $(495 \pm 124 \mathrm{ng} / \mathrm{ml}), \mathrm{ARM}(507 \pm 145 \mathrm{ng} / \mathrm{ml})$ and CNV patients $(523 \pm 100 \mathrm{ng} / \mathrm{ml})$ were not significantly different $(P=0.8)$, as shown in Figure 1 .

\section{Total plasma MMP-9 levels}

The mean plasma MMP-9 levels were significantly higher in ARM $(659 \pm 315 \mathrm{ng} / \mathrm{ml})$ and CNV
$(740 \pm 494 \mathrm{ng} / \mathrm{ml})$ compared to that of control patients $(265 \pm 134 \mathrm{ng} / \mathrm{ml}, P=0.008)$, as demonstrated by

Figure 2.

\section{Discussion}

In this study, plasma MMP-9 levels in AMD subjects were found to be approximately threefold higher than controls. However, there was no significant difference in MMP-9 levels between the ARM and CNV groups. Plasma MMP-2 levels were noted to be similar in the three groups.

There is growing evidence that MMPs play important roles in many disease processes, but most of these enzymes are likely to be active at a local tissue level.

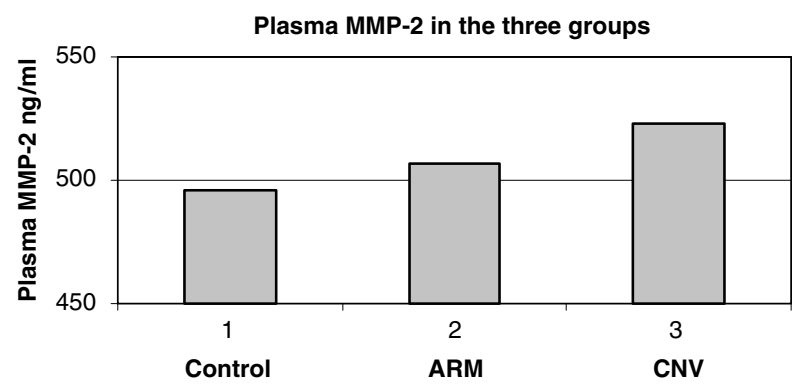

Figure 1 Mean plasma MMP-2 levels in the three groups.

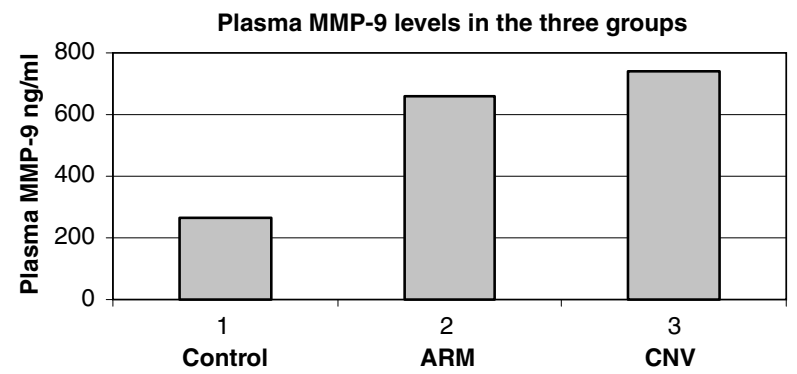

Figure 2 Mean serum plasma MMP-9 levels in the three groups.

Table 1 Characteristics of subjects in the three groups

\begin{tabular}{lcccc}
\hline & ARM $(\mathrm{n}=15)$ & CNV $(\mathrm{n}=18)$ & Controls $(\mathrm{n}=17)$ & P-value \\
\hline Age (years with range) & $74(61-81)$ & $75(62-91)$ & $76(61-81)$ & 0.5 \\
Gender M:F & $5: 10$ & $7: 11$ & 0 & 0.5 \\
Smoker (\%) & 6.6 & 5.5 & 35.3 & 0.7 \\
Hypertension (\%) & 33.3 & 27.7 & 5.9 & 0.7 \\
Myocardial infarction (\%) & 6.6 & 11.11 & 5.9 & 0.6 \\
Angina pectoris (\%) & 0 & 0 & 0 & 0.3 \\
Stroke (\%) & 6.6 & 11.11 & 17.6 & 0.3 \\
Hypercholesterolemia (\%) & 13.3 & & 0.6 \\
\hline
\end{tabular}

Abbreviations: ARM, age-related maculopathy; $\mathrm{CNV}$, choroidal neovascularization. 
Nonetheless, raised plasma MMP-9 levels have been documented in cancer, hepatic and lung diseases and rheumatoid arthritis. ${ }^{17-20}$ Subjects with history of these conditions were excluded from this study. The level of circulating MMP-9 is raised in atherosclerosis. ${ }^{21} \mathrm{No}$ statistically significant differences in clinical data related to atherosclerosis were observed between subjects in the three groups in this study. The levels of plasma MMP-9 also vary in different ethnic groups but all the patients in this cohort were Caucasians. ${ }^{22}$ Therefore, these findings of raised plasma MMP-9 in this study is most probably associated with AMD. There may be other unknown confounding factors body mass index that may influence the results. However, they should be highly associated with MMP-9 to explain these results.

The weakness of this study is that total MMP-2 and MMP-9 (pro and active forms) were measured. All MMPs require activation from precursors to attain enzymatic activity. The antibodies used did not distinguish the active form of these enzymes from their proenzyme forms. Gelatin zymography is a more sensitive assay for detection of both latent and active forms of MMP-2 and MMP-9. In addition, tissue inhibitors of metalloproteinases (TIMPs) dissociate from MMPs during electrophoresis thus preventing TIMPs from inhibiting enzymatic activity. ${ }^{23}$ Therefore, increased MMP-9 immunoreactivity in this study does not necessarily correspond to its augmented enzymatic activity.

Nevertheless, the source of increased MMP-9 in the circulation in AMD patients remains unclear. The contribution of the RPE-BM complex to the circulating MMP-9 is presumably negligible, it is not thought likely that increased MMP-9 occurs as a result of AMD in the first instance. Other important sources of MMPs include astrocytes, neurons, microglia, leukocytes and macrophages. ${ }^{24}$ It is therefore possible that circulating leukocytes may contribute to the increased levels of MMP-9. The recent evidence of raised C-reactive protein (CRP) in intermediate and advanced AMD suggests a state of low-grade inflammation. ${ }^{25}$ Proinflammatory cytokines may also account for macrophage activation and subsequent release of MMP-9. ${ }^{26}$ Furthermore, oxidative stress can result in enhanced expression and activation of MMPs. ${ }^{27}$ Further investigations are needed to address these issues.

The lack of an increase in MMP-2 levels may reflect its more constitutive expression, whereas MMP-9 levels are more responsive to reactive oxygen species and inflammatory cytokines. ${ }^{28}$

In summary, this is the first study that reveals an association of circulating MMP-9 with AMD. It is difficult to make wide-ranging conclusions/assumptions based on these observations in view of the small sample size.
However, this is an important starting point. Larger scale future studies will be required to clarify these findings including the link with systemic inflammatory markers.

\section{References}

1 Vingerling JR, Dielemans I, Hofman A, Grobbee DE, Hijmering M, Kramer CF et al. The prevalence of age-related maculopathy in the Rotterdam study. Ophthalmology 1995; 102: 205-210.

2 Mitchell P, Smith W, Attebo K, Wang JJ. Prevalence of agerelated maculopathy in Australia. The Blue Mountains Eye Study. Ophthalmology 1995; 102: 1450-1460.

3 Klein R, Klein BE, Jensen SC, Meuer SM. The five-year incidence and progression of age-related maculopathy. Beaver Dam Eye Study Ophthalmol 1997; 104: 7-21.

4 Klein R, Klein BE, Linton KL. Prevalence of age-related maculopathy. Beaver Dam Eye Study Ophthalmol 1992; 99: 933-943.

5 Johnson LV, Anderson DH. Age-related macular degeneration and the extracellular matrix. $N$ Engl J Med 2004; 351: 320-322.

6 Ng EW, Adamis AP. Targeting angiogenesis, the underlying disorder in neovascular age-related macular degeneration. Can J Ophthalmol 2005; 40: 352-368.

7 Spraul CW, Lang GE, Grossniklaus HE, Lang GK. Histologic and morphometric analysis of the choroid, Bruch's membrane, and retinal pigment epithelium in postmortem eyes with age-related macular degeneration and histologic examination of surgically excised choroidal neovascular membranes. Surv Ophthalmol 1999; 44: S10-S32.

8 Chong NH, Keonin J, Luthert PJ, Frennesson CI, Weingeist DM, Wolf RL et al. Decreased thickness and integrity of the macular elastic layer of Bruch's membrane correspond to the distribution of lesions associated with age-related macular degeneration. Am J Pathol 2005; 166: 241-251.

9 Guo L, Hussain AA, Limb GA, Marshall J. Age-dependent variation in metalloproteinase activity of isolated human Bruch's membrane and choroid. Invest Ophthalmol Vis Sci 1999; 40: 2676-2682.

10 Kamei M, Hollyfield JG. TIMP-3 in Bruch's membrane: changes during aging and in age-related macular degeneration. Invest Ophthalmol Vis Sci 1999; 40: 2367-2375.

11 Lambert V, Wielockx B, Munaut C, Galopin C, Jost M, Itoh T et al. MMP-2 and MMP-9 synergize in promoting choroidal neovascularization. FASEB J 2003; 17: 2290-2292.

12 Lambert V, Munaut C, Jost M, Noel A, Werb Z, Foidart JM et al. Matrix metalloproteinase-9 contributes to choroidal neovascularization. Am J Pathol 2002; 161: 1247-1253.

13 Mullins RF, Russell SR, Anderson DH, Hageman GS. Drusen associated with aging and age-related macular degeneration contain proteins common to extracellular deposits associated with atherosclerosis, elastosis, amyloidosis, and dense deposit disease. FASEB J 2000; 14: 835-846.

14 Blankenberg S, Rupprecht HJ, Poirier O, Bickel C, Smieja M, Hafner $\mathrm{G}$ et al. AtheroGene Investigators. Plasma concentrations and genetic variation of matrix metalloproteinase 9 and prognosis of patients with cardiovascular disease. Circulation 2003; 107: 1579-1585.

15 Auge N, Maupas-Schwalm F, Elbaz M, Thiers JC, Waysbort A, Itohara $S$ et al. Role for matrix metalloproteinase-2 in oxidized low-density lipoprotein-induced activation of the 
sphingomyelin/ceramide pathway and smooth muscle cell proliferation. Circulation 2004; 110: 571-578.

16 Bird AC, Bressler NM, Bressler SB, Chisholm IH, Coscas G, Davis MD et al. An international classification and grading system for age-related maculopathy and age-related macular degeneration. The International ARM Epidemiological Study Group. Surv Ophthalmol 1995; 39: 367-374.

17 Farias E, Ranuncolo S, Cresta C, Specterman S, Armanasco E, Varela $\mathrm{M}$ et al. Plasma metalloproteinase activity is enhanced in the euglobulin fraction of breast and lung cancer patients. Int J Cancer 2000; 89: 389-394.

18 Chung TW, Kim JR, Suh JI, Lee YC, Chang YC, Chung TH et al. Correlation between plasma levels of matrix metalloproteinase (MMP)-9 /MMP-2 ratio and alphafetoproteins in chronic hepatitis carrying hepatitis B virus. J Gastroenterol Hepatol 2004; 19: 565-571.

19 Ohbayashi H. Matrix metalloproteinases in lung diseases. Curr Protein Pept Sci 2002; 3: 409-421.

20 Tchetverikov I, Ronday HK, Van El B, Kiers GH, Verzijl N, TeKoppele JM et al. MMP profile in paired serum and synovial fluid samples of patients with rheumatoid arthritis. Ann Rheum Dis 2004; 63: 881-883.

21 Kai H, Ikeda H, Yasukawa H, Kai M, Seki Y, Kuwahara F et al. Peripheral blood levels of matrix metalloproteases-2 and -9 are elevated in patients with acute coronary syndromes. J Am Coll Cardiol 1998; 32: 368-372.
22 Tayebjee MH, Lip GY, Blann AD, Macfadyen RJ. Effects of age, gender, ethnicity, diurnal variation and exercise on circulating levels of matrix metalloproteinases (MMP)-2 and -9 , and their inhibitors, tissue inhibitors of matrix metalloproteinases (TIMP)-1 and -2. Thromb Res 2005; 115 205-210.

23 Kleiner DE, Stetler-Stevenson WG. Quantitative zymography: detection of picogram quantities of gelatinases. Anal Biochem 1994; 218: 325-329.

24 Lorenzl S, Albers DS, Relkin N, Ngyuen T, Hilgenberg SL, Chirichigno $\mathrm{J}$ et al. Increased plasma levels of matrix metalloproteinase-9 in patients with Alzheimer's disease. Neurochem Int 2003; 43: 191-196.

25 Seddon JM, Gensler G, Milton RC, Klein ML, Rifai N. Association between C-reactive protein and age-related macular degeneration. JAMA 2004; 291: 704-710.

26 Beuche W, Yushchenko M, Mader M, Maliszewska M, Felgenhauer K, Weber F. Matrix metalloproteinase-9 is elevated in serum of patients with amyotrophic lateral sclerosis. Neuroreport 2000; 11: 3419-3422.

27 Okamoto T, Akaike T, Sawa T, Miyamoto Y, van der Vliet A, Maeda H. Activation of matrix metalloproteinases by peroxynitrite-induced protein S-glutathiolation via disulfide S-oxide formation. J Biol Chem 2001; 276: 29596-29602.

28 Girolamo F, Virgintino D, Errede M, Capobianco C, Bernardini N, Bertossi $\mathrm{M}$ et al. Involvement of metalloprotease-2 in the development of human brain microvessels. Histochem Cell Biol 2004; 122: 261-270. 